\title{
Analysis of a Function Fitting Neural Network based Control Strategy for a Hybrid Energy Storage System applied to Electric-Vehicles
}

\author{
Saket Tirpude ${ }^{1}$, Ankit Bharadwaj ${ }^{2}$, Abhiyan Dubey ${ }^{3}$, R M Holmukhe $^{4}$ \\ ${ }^{1}$ Department of Electrical Engineering, College of Engineering, Bharti Vidyapeeth (Deemed to be) University, \\ India, stirpude1298@gmail.com \\ ${ }^{2}$ Department of Electrical Engineering, College of Engineering, Bharti Vidyapeeth (Deemed to be) University, \\ India, bharadwajankit23@gmail.com \\ ${ }^{3}$ Department of Electronics and Telecommunication Engineering, College of Engineering, Bharti Vidyapeeth \\ (Deemed to be) University, India, abhiyan111@ gmail.com \\ ${ }^{4}$ Department of Electrical Engineering, College of Engineering, Bharti Vidyapeeth (Deemed to be) University, \\ India, rajeshmholmukhe@ hotmail.com
}

\begin{abstract}
One of the crucial aspects of a hybrid energy storage system consisting of super-capacitor (SC) and Li-ion battery is the selection of the control strategy which gives an optimized flow of system parameters. In this paper, a hybrid energy storage system (HESS) for application to electric vehicles (EVs) is modeled using a bidirectional DC-DC converter on Matlab/Simulink. The control system circuit is realized using a proportional-integral (PI) and a function fitting neural network (FNN). A comparative analysis between the two aforementioned control strategies is carried out based upon system parameters such as lithium-ion battery (LI) current, super-capacitor (SC) current, and load voltage. As a result, it is observed that with the FNN control strategy, the system parameters in concern have reduced noise amplitude, distortions, and lesser oscillations in the signals and are stable when compared against the signals produced by PI control strategy. The results from the FNN based control strategy corroborate optimized power quality and performance characteristics, augmenting the overall efficiency and increase in the life cycles of the electric-vehicle (EV).
\end{abstract}

Key words: Hybrid Energy Storage System (HESS), Electric-Vehicle (EV), Super-capacitor (SC), Lithium-ion Battery, Proportional-Integral (PI) Controller, Neural Network (NN), Control Strategy, Energy Management Strategy.

\section{INTRODUCTION}

Electric Vehicles (EVs) are propagating at a meteoric rate with interests ranging from academicians to industries. Much of the rampant increase is attributable to their high efficiency and low environmental impact. Although in many developed nations, EVs are already abundant in the market, the developing nation are including measures to boost the adoption of EVs in their markets. It is also in consensus across the variegated researchers that rectification in the deficiencies are needed to substantially increase their effectiveness against conventional IC-Engine based vehicles, particularly in aspects of economics and battery range.

The excessive pollution due to fossil fuels has paved a way for EVs in which alternate forms of energy sources have been consistently researched and developed [1],[2]. The majority of the energy storage system (ESS) in the EVs constitutes of batteries alone such as Lead-Acid or Lithium-Ion. EVs such as electric scooters, forklifts, golf carts, and e-rickshaws (A common three-wheeled passenger vehicle used in Asia) employ lead-acid batteries more. However, impending electric traction and ESS focuses more on the implementation of LI battery. Nonetheless, in broad terms, batteries have high energy density but an average power density. No doubts that the LI batteries can provide long commutes for EVs, but it is still vulnerable to the range anxiety which an operator of the EV often succumbs to.

Thus, in efforts to resolving these issues, hybrid energy storage systems came into their own. To make electric vehicles efficient to a level where it transcends the IC-Engine based vehicles in respect of higher power, energy densities and long-distance commute, a HESS consisting of a combination of LI batteries and super-capacitors is applied to electric vehicles enabling HESS to being one of the most sought after topics in the research of EVs [3].

DC-DC converters are a pivotal part of a HESS. A lot of research has been carried out on DC-DC converters and many unique designs have been proposed. An efficient DC-DC converter should mainly emphasize on good controllability, 
large power transmission conversion, improved conversion efficiency, and at the same time should be economical with easier manipulation of the control [4].

Energy management strategies become essential for the HESS to achieve maximum efficiency. The poor power quality, noise in the signals and waveform distortion depends heavily on the kind of energy management strategies being put to use. It is vital to opt for an appropriate energy management strategy that optimizes the load voltage, battery current, super-capacitor current such that a smooth waveform which is free from noise and distortion is achieved. Control strategy and in turn the controller also plays an immense role in the e-vehicles, especially for an efficient energy management strategy. For the EV to have an accord with the actual working conditions on the road, many real-time controllers have been proposed in the past. While it is irrefutable that the amalgamation of LI batteries and SC in a HESS makes the system yielding in a sense that control of the HESS becomes efficient, it is also necessary to look into the modifications that can be implemented in the controller which may further increase the performance of the HESS. Modern controllers such as the adaptive rule-based controllers and the comprehensive controllers possess many advantages, some of them being lessening the battery life lost and improved state of charge consumption. The latter configuration is known to have reduced the battery life loss by nearly $3 \%$ and SOC consumption by $1.88 \%$ [5],[6].

In this paper, an attempt to improve the current and voltage signals with a PI-based control strategy is carried out. The implementation of the HESS topology is based on an integrated magnetic structure type DC-DC. The control strategy for the supercapacitor and the li-ion battery pack is implemented through the FNN. The designing is carried and simulated on Matlab/Simulink and the results are compared against a Proportional-Integral (PI) based control system for SC and LI battery pack. The FNN control strategy ameliorates the noise amplitude and distortion in the load voltage, SC current and battery current. This scheme improves the stability, augments the efficiency which results in a long life of the EV.

\section{HYBRID ENERGY STORAGE SYSTEMS}

Contrary to the EVs employing single energy source such as a LI battery or a Lead-acid battery, an EV with two different energy sources namely SC and LI battery working as a cohesive system makes the starting of the EV quicker without waiting for the pre-heating of batteries, gives a better EV mileage, pacifies the range anxiety and makes the vehicle operation much more efficient under very low temperature and harsh conditions. The peak currents drawn from the battery (especially when the battery SOC is low) puts a lot of stress on the battery and in the long run decreases the battery life extensively. A HESS design implemented in an EV increases the efficiency of the system by great margins and increases the battery life [7].

HESS deals with attending to two typical cases i.e. the high-power demand and the high-energy storage. The former resolves the issues of rapid load fluctuations, transients and is known for a fast response time, whereas the latter is characterized by a lower self-discharge rate and mostly attends to providing consistent energy at a vast interval of time.

HESS possesses many advantages some of them being coupling SC and LI battery pack and functioning the LI battery pack at high efficient operating points, making the flow of system parameters optimized and boosting the total system efficiency. It also aids in reducing the dynamic losses and stresses of the LI battery which leads to enhanced storage and system life. From a monetary point of view, the use of HESS in EVs is economical as the total investment costs are lower when compared against the conventional ESS consisting of LI battery alone [8].

Many different schemes have been applied to couple the $\mathrm{SC}$ and Li-ion battery. Direct DC coupling of two sources is the more renowned as it is cost-effective and simple in structure. Figure 1 shows the direct DC coupling scheme.

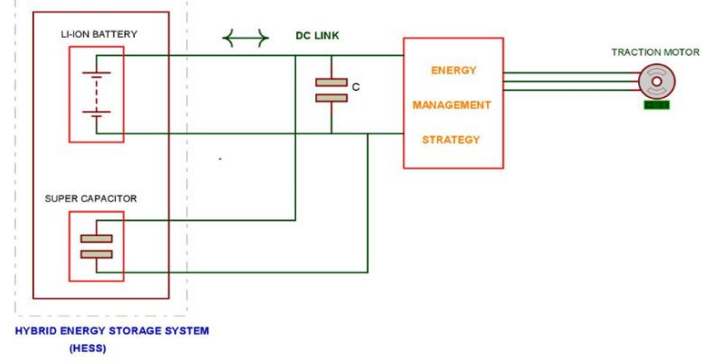

Figure 1: Coupling of SC and Lithium-ion battery via a DC link.

The major disadvantage with this scheme is the inability to modulate power flow and energy management leading to a wanting employment of the energy sources. Other coupling schemes include using a single bidirectional DC-DC converter or two DC-DC converters. The former scheme can be connected to either of the two energy sources. The only disadvantage accompanying using a bidirectional DC-DC converter is the fluctuations in the voltage levels. The use of separate DC-DC converters for each source is the most efficacious coupling architecture which operates the increased power density source at a broader voltage band optimizing the available energy storage capacity to the best of its ability. However, this scheme is inhibited by its complexity and exorbitant prices [9],[10]. Figures 2 and 3 show the two schemes below. 


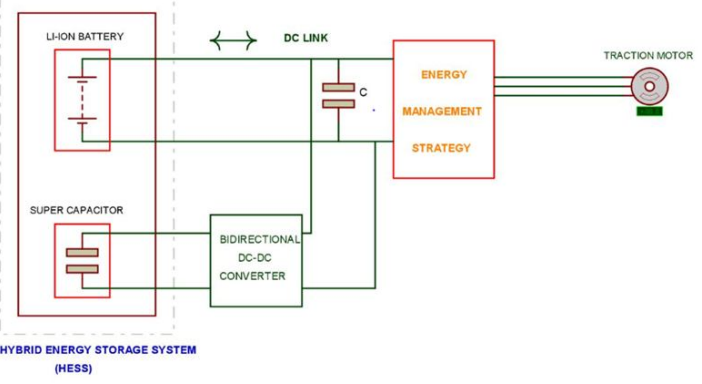

Figure 2: Coupling of SC and Lithium-ion battery by a single Bidirectional DC-DC converter.

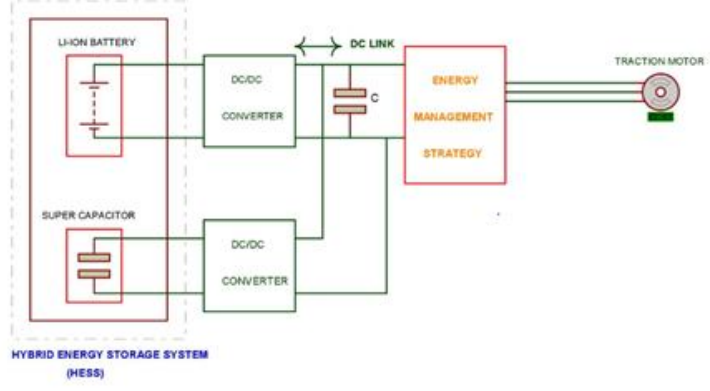

Figure 3: Coupling of SC and Li-ion battery by two separate DC-DC converters.

The operation of EVs incorporating lead-acid batteries is inhibited at low-temperatures owing to the freezing of the electrolyte. This issue is somewhat mitigated by EVs incorporating LI batteries which can still manage to operate at comparative temperatures. Most of the research work about HESS emphasizes more on the role of HESS in power capability and improving the efficiency of the system but undermine its performance at different temperatures. HESS consisting of a lithium-ion capacitor and HESS consisting of a high-power lithium-ion battery can operate smoothly at a temperature range of $-10{ }^{\circ} \mathrm{C}$ and $-20^{\circ} \mathrm{C}$, while increasing the power capability and drive range substantially. This ensures that different HESS schemes in EVs overcome any barriers relating to dependence on temperature [11],[12].

\section{CONTROL STRATEGY FOR HYBRID ENERGY STORAGE SYSTEM}

To provide a stable load voltage and current through the SC and the battery pack, it is necessary to choose a suitable control and energy management strategy which is most efficient in delivering the desired outputs. Many cascaded voltage and current controllers have in the past, sufficed in providing the desired voltages and currents. These controllers were based on a Proportional-Integral-Derivative (PID) or Proportional-Integral (PI) based control algorithms and were able to optimize the system parameters up to the nearest mark of the desired levels. However, many other alternatives have also been referred to in the research literature in recent years. These include some adaptive and intelligent control strategies such as fuzzy logic and artificial neural network-based controllers [13],[14],[15],[16].

In this paper, we limit our scope to analyze the responses from the PI-based controller and the FNN based controllers, discuss the pros and cons of each and give a detailed comparative analysis of them in the later sections.

\subsection{PI-Based Control Strategy}

The PI based controllers, is one of the most renowned control strategies implemented in various HESS applications, whether it be HESS's application to renewable energy or EVs. The PI controllers act on the errors between the desired signal and the controlled signal, hence possessing no superfluous measurements for internal states. The transfer function $[\mathrm{G}(\mathrm{s})]$ of a PI-based controller is given by;

$$
\mathrm{G}(\mathrm{s})=\frac{\mathrm{sK}(\mathrm{p})+\mathrm{K}(\mathrm{i})}{\mathrm{s}}
$$

Where; K(p), K(i) are Proportional and Integral constants.

A controller signal $\mathrm{x}(\mathrm{t})$ is fed inside the system whose equation is shown below;

$$
\mathrm{x}(\mathrm{t})=\mathrm{x}(\mathrm{b})+\mathrm{K}_{\mathrm{c}} \mathrm{e}(\mathrm{t})+\underline{\mathrm{K}}_{\Gamma_{\mathrm{c}}} \int_{\mathrm{a}}^{e} e(\mathrm{t}) d t
$$

The value $\mathrm{x}(\mathrm{b})$ is a constant set to accommodate to the value of $x(t), K c$ is the controller gain and $\Gamma_{c}$ is the time constant. $e(t)$ is the error signal which is the aberration recorded between the desired point (DP) and process variable (PV) given by the following equation;

$$
\mathrm{e}(\mathrm{t})=\mathrm{DP}-\mathrm{PV}
$$

For the simulation in this paper, we employed a discrete time domain to realise the PI controller. For discrete sampling periods, an equation in the discrete form is required to measure the approximations of the error signals. This equation is shown below;

$$
\mathrm{x}(\mathrm{t})=\mathrm{x}(\mathrm{b})+\frac{\mathrm{Kc} e(\mathrm{t})}{\Gamma_{\mathrm{c}}}+\mathrm{Kc} \sum_{\mathrm{i}=\mathbb{1}} \operatorname{eic} \mathrm{t} t
$$

More measurements of the internal states would mean increased number of sensors which will require more devices and hence higher maintenance cost. Thus, PI controllers offer an economical solution on this front which is its biggest advantage. The architecture is simple, so the controllability of the system becomes easier, robust and efficient. However, today where the smart energy systems are able to adapt to changing environments so that real-time operation becomes feasible, the PI controller fails to accustom to the real time changes. The figure 4 below shows block diagram of the PI controller used as a voltage and current controller for SC. Figure $5 \& 6$ show a similar voltage and current controller for LI battery pack and SC. 


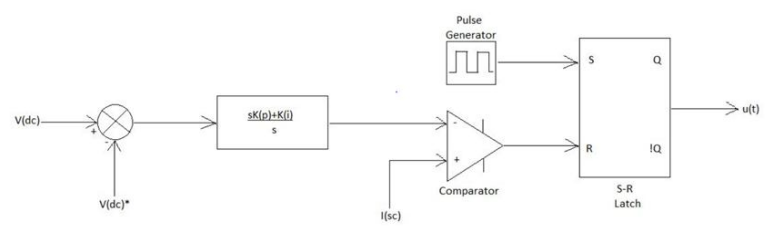

Figure 4: Block diagram of PI-based control strategy used for voltage and current control.

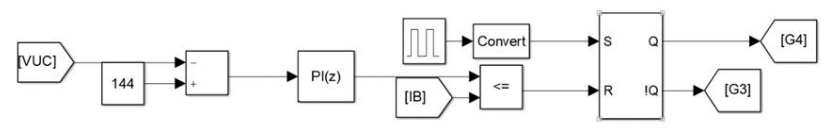

Figure 5: PI-based voltage and current controller for SC.

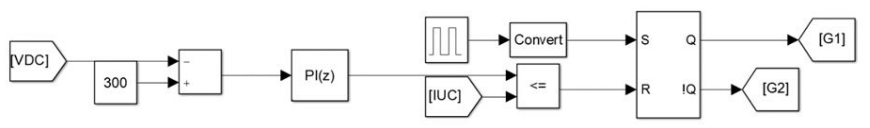

Figure 6: PI-based voltage and current controller for LI battery.

\subsection{Function Fitting Neural Network (FNN)-Based Control Strategy}

Neural networks $(\mathrm{NN})$ have for long been proposed for use as a control strategy in HESS. This can be mostly attributed to the feature that $\mathrm{NN}$ can adapt to different changing inputs. In past, many different hybrid controllers such as a math function-based controller with neural networks have been proposed and the results show that the switching between SC and battery can be eliminated with such a hybrid controller [17].

The FNN controllers are data-driven algorithms that are similar to how the human brain works. The information is carried out through nodes with the help of small computational elements called neurons. The FNN system works in different layers and inherently forms layers (also called multilayer perceptron) to produce the desired output which is stable. The multilayer perceptron consists of three layers- an input layer, a hidden layer, and the output layer. The input layer takes the values of the independent variables and passes on to the nodes of the hidden layer. The information received by the hidden layer is processed and passed on to the output layer. The same process is carried out for each node. The node receives information (Un) from multiple nodes of the previous layer which carries their unique weight $(\mathrm{W})$ value which is summed at the node with a unique bias (B) number [18].

This summation is carried forward and processed with the help of an activation function to the output. The output received is termed as the predicted value of the dependent variable which is being compared by the network with the actual values. If the predicted value doesn't match with the actual value the process is carried out again with different weight values. In this paper, our responses were sufficed by a 2-layer neural network which is shown in figure 7 below. The major advantage with $\mathrm{NN}$ is that it generates the best possible result without needing to redesign the output criteria. Figures 8 and 9 show the FNN-based voltage and current controller for SC and battery.

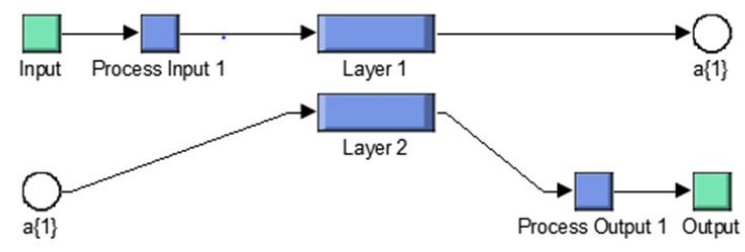

Figure 7: Two-layer FNN used in the paper and simulated on Matlab/Simulink

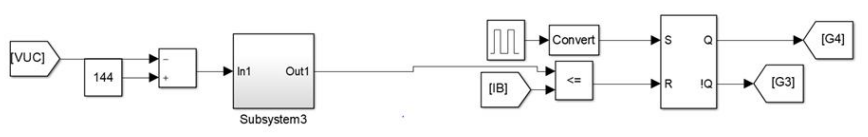

Figure 8: FNN-based voltage and current controller for super-capacitors.

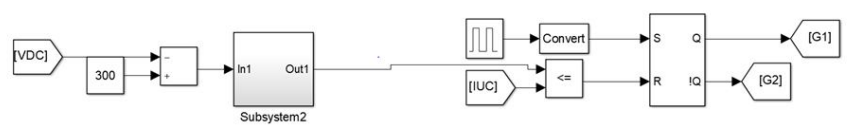

Figure 9: FNN-based voltage and current controller for lithium-ion battery.

\section{METHODOLOGY}

In this paper, the schematic of the HESS is modeled in Matlab/Simulink. Figure 9 shows the block diagram of the HESS being modeled and constitutes of different power electronic devices as shown in figure 9. To convert the Alternating Current (AC) Mains into Direct Current (DC), a universal bridge rectifier is used. With every switching device in the bridge rectifier, a series RC snubber circuit is connected in parallel. For switching action, four IGBTs (T1 to T4) are used. The integrated magnetic structure is implemented by using a mutual inductance (M) composed of two self-inductance L1 and L2. This paper chooses a LI battery and an SC for the energy storage and instantaneous peak power supply. Table 1 and Table 2 show the parameters of each of them below. 
Saket Tirpude et al., International Journal of Emerging Trends in Engineering Research, 8(7), July 2020, 2955 - 2962

Table 1: Basic parameters of a LI Battery

\begin{tabular}{|l|l|}
\hline Parameter & Value \\
\hline Rated Capacity (Ah) & 70 \\
\hline Nominal Voltage (Volts) & 144 \\
\hline Initial State of Charge $(\%)$ & 100 \\
\hline Internal Resistance $(\mathrm{Ohms})$ & 0.020571 \\
\hline Operating Temperature $\left({ }^{\circ} \mathrm{C}\right)$ & $-20 \sim 70$ \\
\hline
\end{tabular}

Table 2: Parameters of the SC

\begin{tabular}{|l|l|}
\hline Parameter & Value \\
\hline Rated Capacitance $(\mathrm{F})$ & 0.2 \\
\hline Rated Voltage $(\mathrm{V})$ & 125 \\
\hline $\begin{array}{l}\text { Equivalent DC Series } \\
\text { Resistance (Ohms) }\end{array}$ & $8.9^{*} 10^{-3}$ \\
\hline Number of Series Capacitors & 20 \\
\hline Number of Parallel Capacitors & 1 \\
\hline Operating Temperature $\left({ }^{\circ} \mathrm{C}\right)$ & 25 \\
\hline Charge Current $(\mathrm{A})$ & 10 \\
\hline
\end{tabular}

The battery and the SC provide the power supply to the traction motor according to the different modes of operation. The traction motor used in the scheme is a wound type DC Motor. The control strategies are separately used for the battery and the SC. As we compare two different types of control strategies in this study, we initially use and implement the HESS with a PI-based SC and battery pack voltage and current controller. The PI controllers are functioned in a discrete-time domain. To actuate and bolster the improvements in the system, the PI controlled strategy is then replaced with a 2-Layer FNN. The comparison and results of the two different control strategy simulations are discussed in the subsequent sections. Table 3 shows the different modes of operation of the DC-DC converter and the power flow in the HESS being implemented. Figure 10 shows the block diagram of the HESS modeled in this paper.

Table 3: Mode of operations of the DC-DC Converter*.

\begin{tabular}{|l|l|l|l|}
\hline Function & $\begin{array}{l}\text { Source of } \\
\text { Power }\end{array}$ & $\begin{array}{l}\text { Flow of } \\
\text { Power }\end{array}$ & $\begin{array}{l}\text { Mode of } \\
\text { Operation of } \\
\text { the DC-DC } \\
\text { Converter }\end{array}$ \\
\hline Charging mode & AC Mains & $\begin{array}{l}\text { LI Battery } \\
\text { and SC }\end{array}$ & Buck \\
\hline $\begin{array}{l}\text { Constant speed } \\
\text { mode }\end{array}$ & LI Battery & DC Motor & Boost \\
\hline $\begin{array}{l}\text { Acceleration } \\
\text { mode }\end{array}$ & SC & DC Motor & Boost \\
\hline $\begin{array}{l}\text { Braking mode } \\
\text { Braking } \\
\text { Energy }\end{array}$ & $\begin{array}{l}\text { LI Battery } \\
\text { and SC }\end{array}$ & Buck \\
\hline $\begin{array}{l}\text { SC charging } \\
\text { mode }\end{array}$ & LI Battery & $\begin{array}{l}\text { SC and } \\
\text { DC Motor }\end{array}$ & Buck/Boost \\
\hline
\end{tabular}

*Source- A Novel Design of Hybrid Energy Storage System for Electric Vehicles, Xiangyang Xia1,2*, Xinxin Zhao1, Heqing Zeng1, and Xiaoyong Zeng1 (1. College of Electrical and Information Engineering, Changsha University of Science and Technology, Changsha 410004, China.

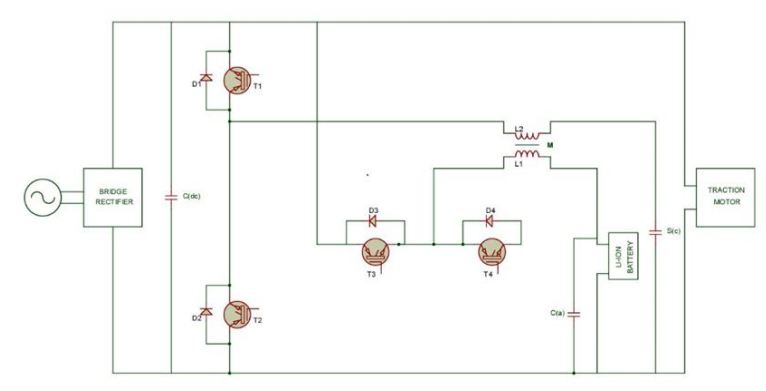

Figure 10: Block diagram of the modelled HESS for EV.

\section{RESULT}

The simulation of the HESS for EV was operated on Matlab/Simulink and was evaluated for its dynamic performance. The simulation with PI controller was implemented first and its results were obtained. Then, the simulation with Neural Network was carried out and tested for system parameters. The two control strategies were mainly compared against three parameters i.e. battery current, super-capacitor current, and load voltage. The schematic of the HESS model applied to EVs which was developed for simulation is shown in figure 11 .

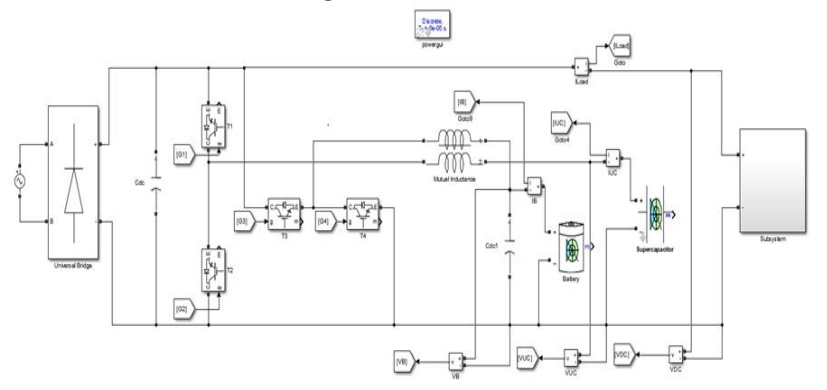

Figure 11: Schematic of the simulation model developed on Matlab/Simulink.

The simulation results of the PI control strategy are shown in figure 12 below. The battery current, SC current, and load voltage are assessed for their stability and response time. Distortion, oscillations, noise, and ripples are observed in the output signals. The battery current is smoother than the SC current where the instantaneous rise edges pester the signal.

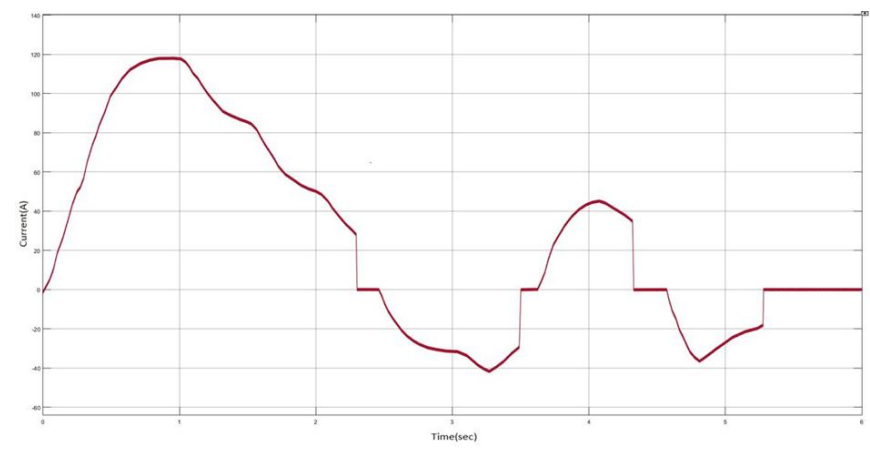

(a). Current response from the lithium-ion battery of the modeled HESS. 


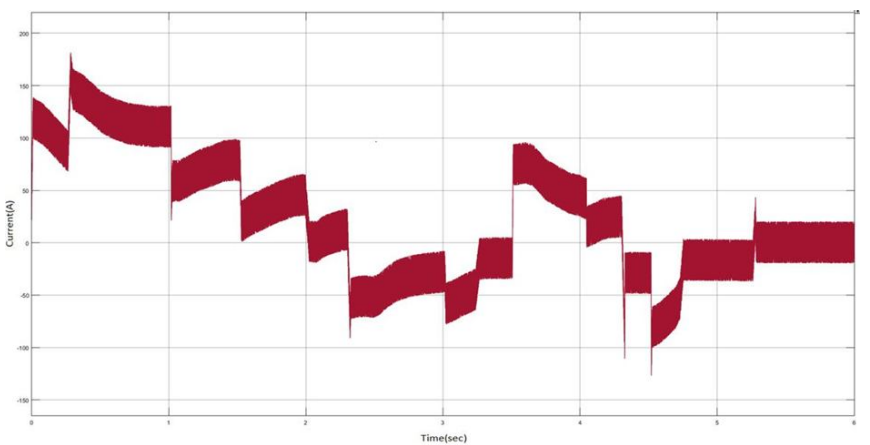

(b). Current response from the $\mathrm{SC}$ of the modelled HESS.

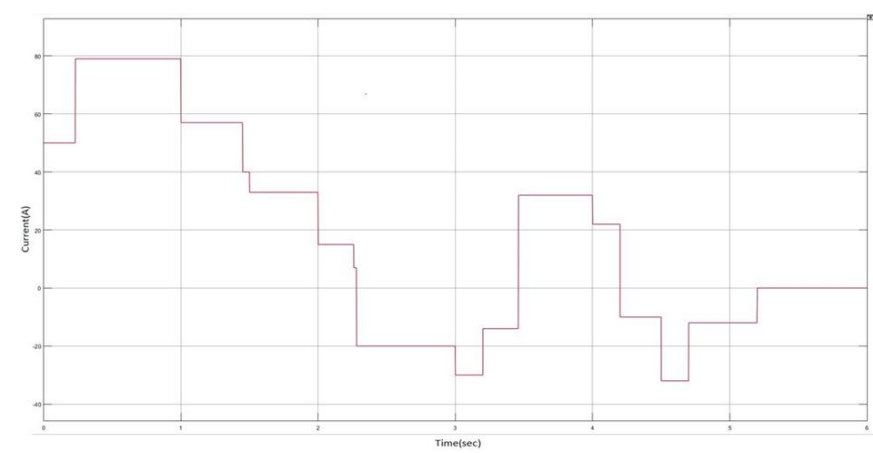

(c). Load Current response of the modelled HESS.

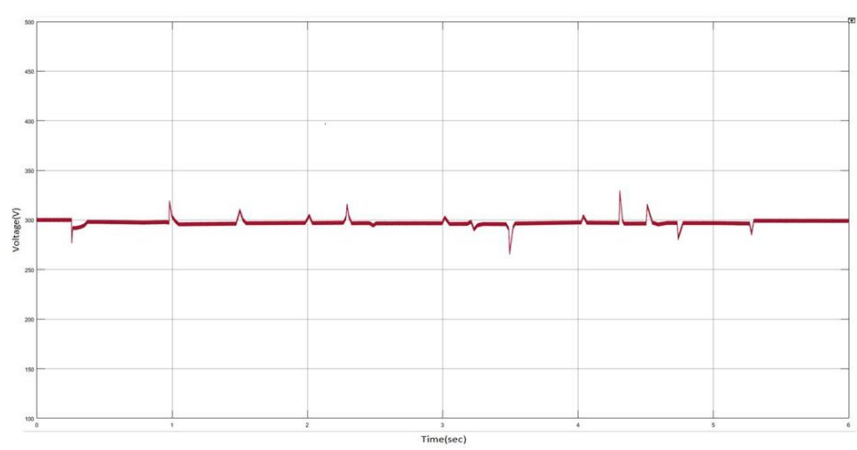

(d). Load Voltage response of the modelled HESS.

Figure 12: Simulation results of the modelled HESS applied on EVs with PI Controller.

The results from the simulation of the modeled HESS applied on EVs with an FNN based control strategy is shown in figure 13 below. This simulation result is also scrutinized for battery, SC, load currents, and load voltage respectively. In this case, responses recorded show the same behaviour as obtained with the PI controller, but with much more reduction in the amplitude of noise and distortion. Oscillations are seen with this control strategy as well, but are reduced by a great margin when compared against the former control strategy.

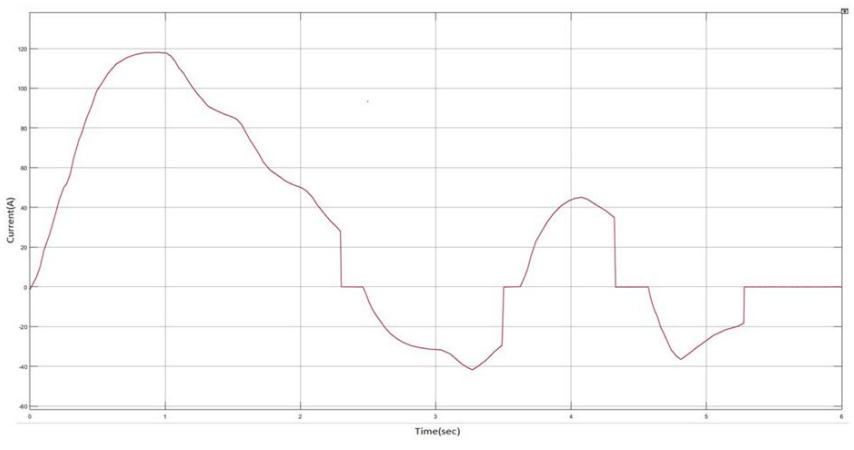

(a). Current response from the lithium-ion battery of the modelled HESS.

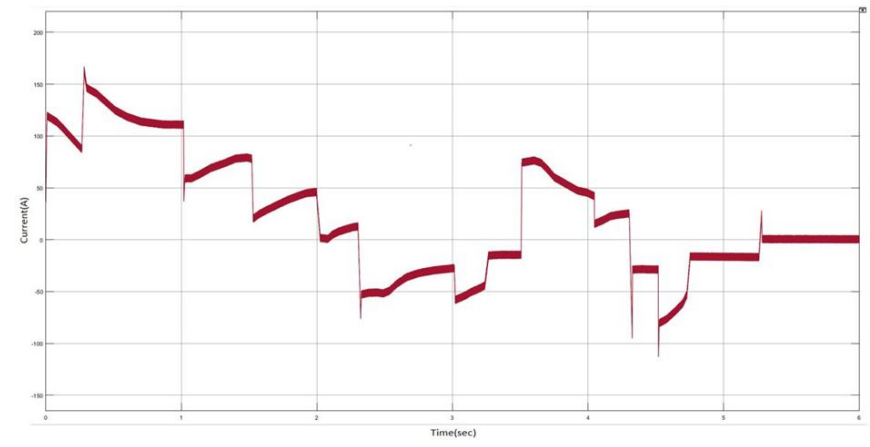

(b). Current response from the SC of the modelled HESS.

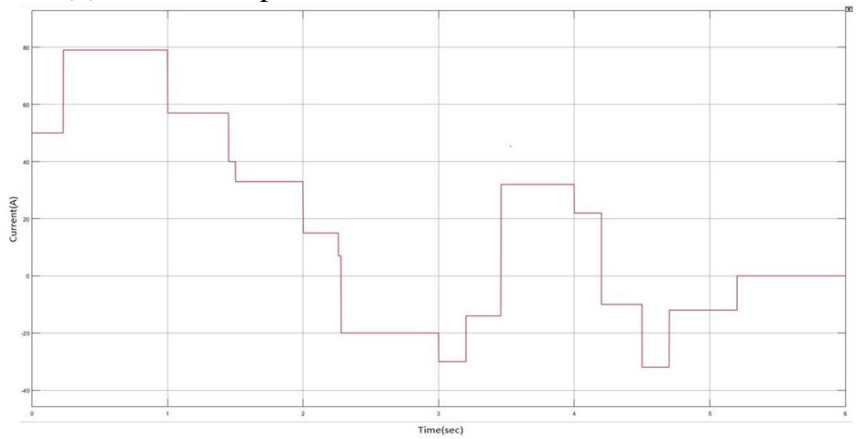

(c). Load Current response of the modelled HESS.

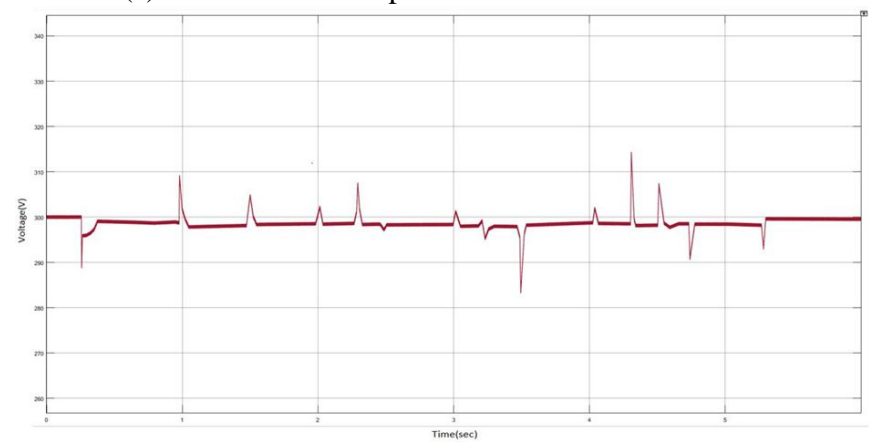

(d). Load Voltage response of the modelled HESS.

Figure 13: Simulation results of the modelled HESS applied on EVs with FNN based controller.

As seen from the simulation results of the two control strategies, the responses from the FNN controllers are smooth. The oscillations and the distortions in the current and voltage responses are lesser than the responses obtained from the PI controller. The close comparative analysis between PI and FNN control strategies for system parameters such as battery current, SC current and load voltage are studied for 
particular time intervals and are shown in figure 14, figure 15 and figure 16 below.

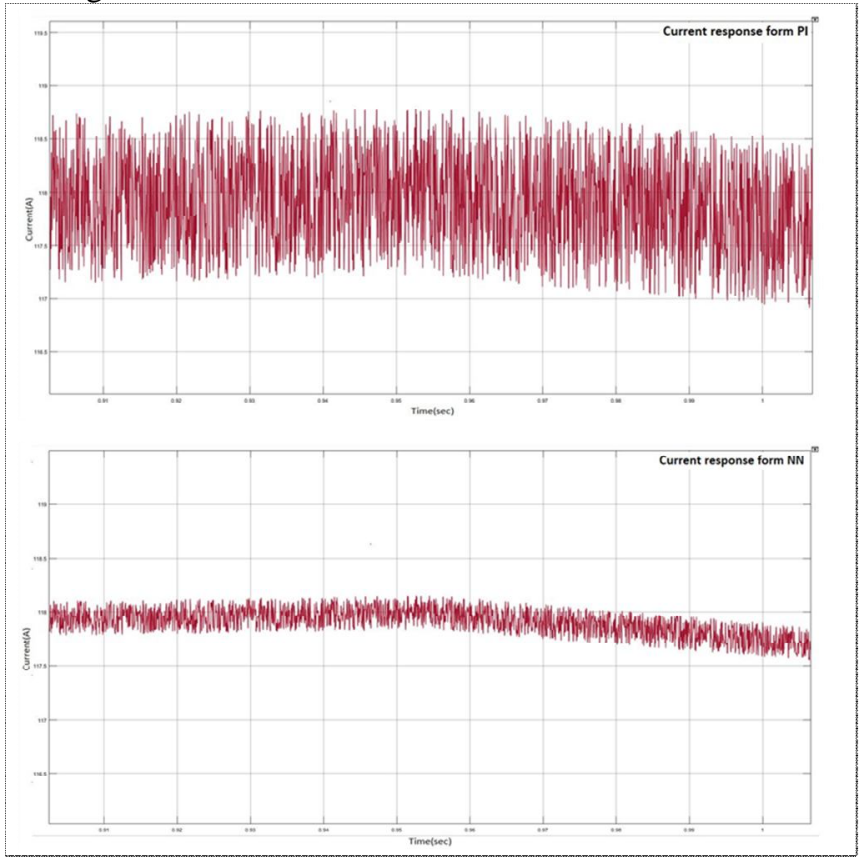

Figure 14: Graph comparing battery current responses from PI and FNN based controllers.

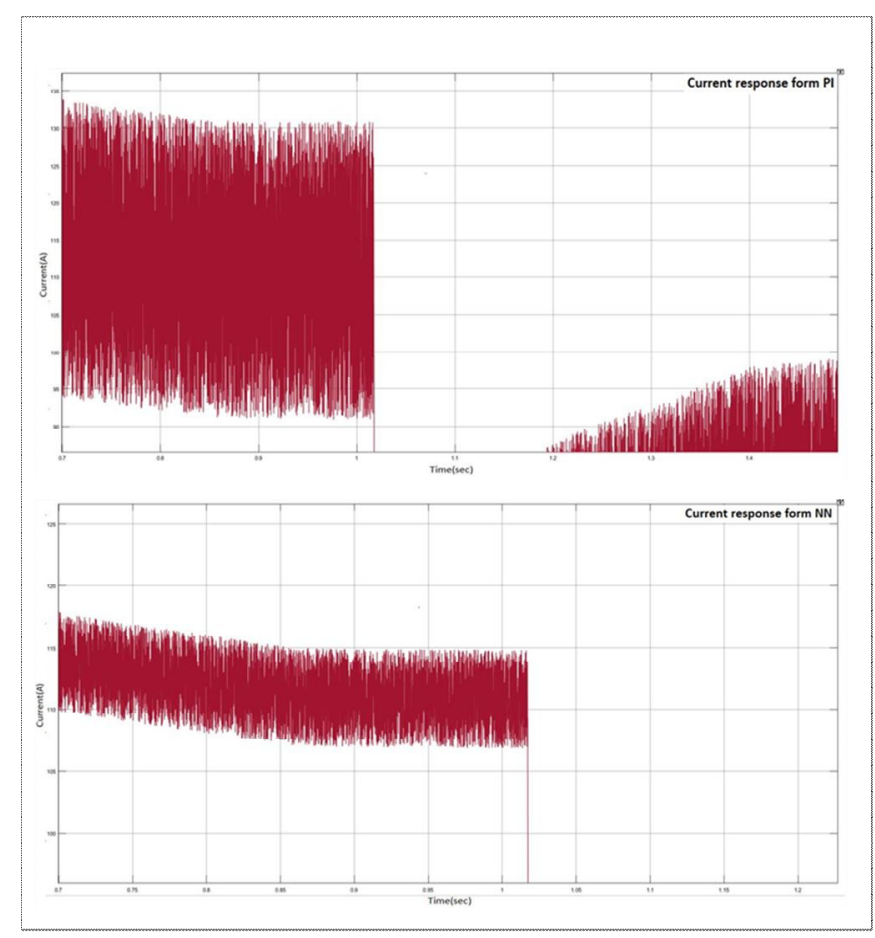

Figure 15: Graph comparing SC current responses from PI and FNN based controllers.

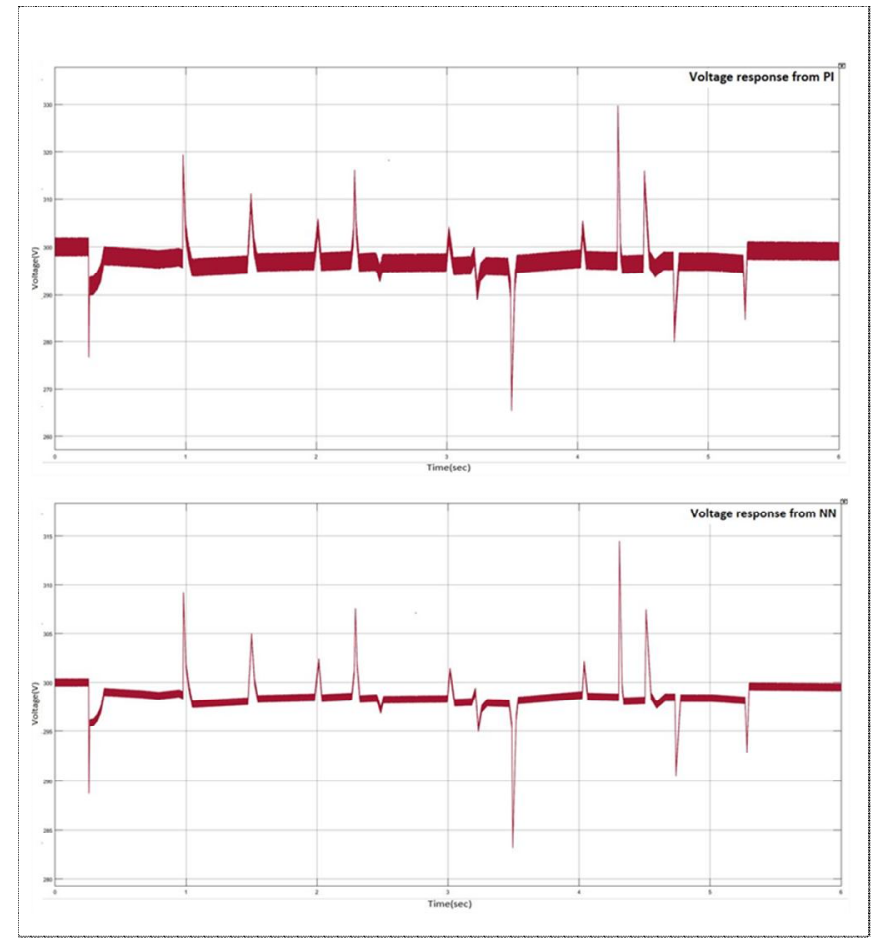

Figure 16: Graphs comparing load voltage responses from PI and NN based controllers.

The battery current for both control strategies is assessed for a common time interval i.e. from $\mathrm{T}=0.90 \mathrm{sec}$ to $\mathrm{T}=1 \mathrm{sec}$. The desired battery current of $118 \mathrm{~A}$ is observed in both cases. In the case of the battery current response from PI controller, the highest positive value of the perturbation being $\sim 118.75 \mathrm{~A}$ and lowest being $\sim 117 \mathrm{~A}$ (Note: Both values considered are nearest approximations). With the FNN control strategy, the battery current output of $118 \mathrm{~A}$ is achieved with significantly reduced perturbations. The SC current responses from both control strategies are shown in figure 15. The SC current responses are observed for a common time interval of $\mathrm{T}=0.7$ sec to $\mathrm{T}=1 \mathrm{sec}$. Again, it can be seen from figure 15 that the response with the FNN control strategy is much improved and stable. The load voltage profile for both strategies are shown in figure 16 for time interval $\mathrm{T}=0 \mathrm{sec}$ to $\mathrm{T}=6 \mathrm{sec}$. For the PI control strategy, it is evident from the recorded response chart that the noise amplitude in the positive side reaches a maximum of $330 \mathrm{~V}$ at $\mathrm{T}=4.4 \mathrm{sec}$ and $266 \mathrm{~V}$ in the negative side at $\mathrm{T}=3.5 \mathrm{sec}$. The noise amplitude with FNN control strategy at $\mathrm{T}=4.4 \mathrm{sec}$ is $314 \mathrm{~V}$ at the positive side, whereas it reaches $284 \mathrm{~V}$ at $\mathrm{T}=3.5 \mathrm{sec}$ at the negative side. Hence, the noise amplitude with the FNN control strategy is improved. From the figures, it is clear that the oscillations, distortions are reduced with FNN controllers, and the noise amplitude in the load voltage is also brought down to 315 when compared against the PI controllers making the system parameter signals smooth and table. Both control strategies did not have any discrepancies when the load current was scrutinized. Reduction in the anomalies and perturbations in the signals will increase the vehicle battery life and its performance efficiency is sure to increase by a great margin. 
Saket Tirpude et al., International Journal of Emerging Trends in Engineering Research, 8(7), July 2020, 2955 - 2962

\section{CONCLUSION}

With keeping in mind the future scope of the EV that it is able to adapt to real-time errors and its efficiency is increased, control strategy should be diligently selected. The Neural Network based control strategy has been scrutinized in this study to improve the signals obtained from the PI-based controller. The performance characteristics of the HESS is better with the FNN control strategy. The FNN-based control strategy transcended the PI-based control strategy by reducing the distortion and amplitude of the noise signals. The FNN controller is also able to nullify the effects of load disturbances and adapts to continuous changing inputs which makes it more advantageous for real-time applications. We conclude that the FNN based control strategy instils waveform that are smooth, decrease the error signals to a modicum and ultimately increases the efficiency of the hybrid energy storage system applied to an EV which in turn leads to increased lifespan.

\section{REFERENCES}

[1] Zhikang Shuai, Chao Shen, Xin Yin, Xuan Liu, John Shen, Fault analysis of inverter-interfaced distributed generators with different control schemes. IEEE Transactions on Power Delivery, DOI: 10. 1109/TPWRD.2717388., 2017

[2] Zhikang Shuai, Yingyun Sun, Z. John Shen, Wei Tian, Chunming Tu, Yan Li, Xin Yin, Microgrid stability: classification and a review. Renewable and Sustainable Energy Reviews, Vol.58, Feb. 2016. pp. 167-179 https://doi.org/10.1016/j.rser.2015.12.201

[3] A. Santucci, A. Sorniotti, and C. Lekakou, Power split strategies for hybrid energy storage systems for vehicular applications. J. Power Sources, Vol. 258, No.14, 2014, pp.395-407,

[4] Xia, Xiangyang \& Zhao, Xinxin \& Zeng, Heqing \& Zeng, Xiaoyong. (2018). A novel design of hybrid energy storage system for electric vehicles. Chinese Journal of Electrical Engineering. Vol. 4, 2018, pp 45-51. 10.23919/cjee.2018.8327370.

[5] Q. Li, W. Chen, Y. Li, S. Liu, and J. Huang, Energy management strategy for fuel cell/battery/ultracapacitor hybrid vehicle based on fuzzy logic. Int. J. Electr. Power Energy Syst., vol. 43, no. 1 , Dec 2012, pp. 514-525

[6] Hu, Jianjun \& Jiang, Xingyue \& Jia, Meixia \& Zheng, Yong. (2018). Energy Management Strategy for the Hybrid Energy Storage System of Pure Electric Vehicle Considering Traffic Information. Applied Sciences. 8. 1266. 10.3390/app8081266.

[7] Barcellona, S., Piegari, L. and Villa, A., Passive hybrid energy storage system for electric vehicles at very low temperatures. Journal of Energy Storage, 25, p.100833, 2019

[8] Sharma Meera, Nijhawan Parag, Sinha Amrita. (2019). Role of Battery Energy Storage System in Modern Electric Distribution Networks - A Review.
International Journal of Advanced Trends in Computer Science and Engineering, Vol. 8, No.3, June 2019, pp. 443-450.

https://doi.org/10.30534/ijatcse/2019/18832019

[9] Tao H, Duarte JL, Hendrix M. Multiport converters for hybrid power sources. PESC Rec. - IEEE AnnualPower Electron. Spec. Conf.; 2008. pp.3412-3418. doi:10.1109/PESC.2008.4592483.

[10] Bocklisch, Thilo. (2015). Hybrid Energy Storage Systems for Renewable Energy Applications. Energy Procedia. 73. 103-111. 10.1016/j.egypro.2015.07.582.

[11]P. Keil and A. Jossen, Improving the Low-Temperature Performance of Electric Vehicles by Hybrid Energy Storage Systems, IEEE Vehicle Power and Propulsion Conference (VPPC), Oct. 2014, pp.1-6.

https://doi.org/10.1109/VPPC.2014.7007087

[12] Keil, Peter \& Englberger, Matthias \& Jossen, Andreas. (2015). Hybrid Energy Storage Systems for Electric Vehicles: An Experimental Analysis of Performance Improvements at Subzero Temperatures. IEEE Transactions on Vehicular Technolog, 2015, 10.1109/TVT.2015.2486040.

[13] M. E. Choi, S. W. Kim, and S. W. Seo, Energy management optimization in a battery/supercapacitor Hybrid energy storage system, IEEE Trans. Smart Grid, Vol. 3, No. 1, Mar. 2012, pp. 463-472,

[14]Z. Yu, D. Zinger, and A. Bose, An innovative optimal power allocation strategy for fuel cell, battery and supercapacitor hybrid electric vehicle,J. Power Sources, Vol. 196, No. 4, Feb. 2011, pp. 2351-2359,

[15]Zhikang Shuai, Yang Hu, Yelun Peng, Chunming Tu, Z. John Shen, Dynamic stability analysis of synchronverter-dominated microgrid based on bifurcation theory, IEEE Transactions on Industrial Electronics, Vol. 64, No. 9, Sep. 2017, pp. 7467-7477. https://doi.org/10.1109/TIE.2017.2652387

[16] Q. Li, W. Chen, Y. Li, S. Liu, and J. Huang, Energy management strategy for fuel cell/battery/ultracapacitor hybrid vehicle based on fuzzy logic. Int. J. Electr. Power Energy Syst., Vol. 43, No. 1, Dec. 2012, pp. 514-525.

[17] Katuri R., Gorantla S. (2019). Design and simulation of a controller for a hybrid energy storage system based electric vehicle, Mathematical Modelling of Engineering Problems, Vol. 6, No. 2, pp. 203-216. https://doi.org/10.18280/mmep.060208

[18] Aaron Don M. Africa, Francis Xavier Asuncion, Janos Lance Tiberio, Raymund Miguel Francisco A. Munchua, Sensor-based Traffic Control Network with Neural Network Based Control System. International Journal of Advanced Trends in Computer Science and Engineering. Vol. 8, No.4, August 2019, pp 983-989. https://doi.org/10.30534/ijatcse/2019/01842019 